\title{
Temporal determinants of occasion setting in feature-positive discriminations
}

\author{
PETER C. HOLIAAND \\ University of Pittsburgh, Pittsburgh, Pennsylvania
}

\begin{abstract}
Three experiments examined the acquisition and transfer of Pavlovian feature-positive discriminations $(\boldsymbol{X A}+, \boldsymbol{A}-)$ in rat subjects. To identify the nature of the associations formed in those discriminations, the form of the conditioned responses (CRs) was examined. If the feature, $X$, and common element, $A$, cues started and ended together on $X A$ compoud trials, associations between $X$ and the food unconditioned stimulus (US) were acquired. If the onsets and/or terminations of $X$ preceded those of $A, X$ acquired the ability to set the occasion for responding to $A$, that is, $A$ evoked CRs only on $X A$ compound trials. The acquisition of occasion setting was favored when (1) the onset of $X$ preceded that of $A,(2)$ the interval between $X$ and $A$ and/or the US was relatively long, and (3) the termination of $X$ occurred prior to the onset of $A$. The occasion-setting power of $X$ was fairly specific to $A$ : $X$ did not modulate responding evoked by another cue that had been first trained and then extinguished or by a cue that had been paired with the US only a few times. However, $X$ did enhance responding to a cue that had been a common element in another, identical feature-positive discrimination. That transfer was somewhat greater if the $X$ and $\boldsymbol{A}$ terminated together than if $\boldsymbol{X}$ terminated prior to the onset of $\boldsymbol{A}$. Implications for theories of stimulus control in Pavlovian conditioning are discussed.
\end{abstract}

In a feature-positive discrimination, a compound stimulus $(X A)$ is reinforced, but one of its elements $(A)$ is separately nonreinforced (Jenkins \& Sainsbury, 1969). Recently, I have presented considerable data that suggested that rats solve feature positive discriminations in different ways, depending on the arrangement of $X$ and $A$ within the $X A$ compound (e.g., Holland, 1983, 1985; Ross \& Holland, 1981). Specifically, I have claimed that in feature-positive discriminations that involve simultaneous $X A$ compounds, $X$ elicits a conditioned response (CR) as a consequence of associations between representations of $X$ and the unconditioned stimulus (US), but in discriminations that involve serial, $X \rightarrow A$ compounds, $X$ acquires the ability to modulate the action of an association between $A$ and the US. Thus, $X$ "sets the occasion" (Moore, Newman, \& Glasgow, 1969; Skinner, 1938) for responding to $A$ that is based on the $A$-US associations.

Ross and Holland (1981) examined the acquisition of feature-positive discriminations in a conditioning preparation in which rats display two behaviors, rear and magazine, in the presence of visual cues for food, but another behavior, head jerk, during auditory signals for food. When simultaneous compounds were presented in training, the form of the rats' behavior to those compounds was determined by the predictive feature. That is, when an auditory + visual compound was reinforced and the auditory cue alone was nonreinforced, the compound

This research was supported in part by Grant MH-37371 from the National Institute of Mental Health. I thank Jenny Lamarre, James Petrick, and Mauricio Suarez for their technical assistance.

Correspondence should be addressed to Peter Holland, who is now at the Department of Psychology, Duke University, Durham, NC 27706. came to evoke rear and magazine behaviors and the auditory cue evoked no CR, as would be anticipated if the visual feature controlled responding by virtue of simple associations between it and the unconditioned stimulus (US). However, when a serial, visual $\rightarrow$ auditory compound was reinforced and the auditory cue alone was nonreinforced, the rats acquired head-jerk behavior to the auditory cue within the serial compound, as if the CR was the consequence of associations between the auditory common-element cue and food. Thus, in the serial discrimination, the visual feature enabled, or set the occasion for, the auditory-cue-food relation.

The experiments reported here attempted to determine what differences between the serial and simultaneous compounds used in Ross and Holland's (1981) experiments were critical to the establishment of occasion setting. The serial compounds that were used in Ross and Holland's experiments involved both onset and termination asynchrony of $X$ and $A$. That is, the feature both began before the onset of the common element and terminated before the common element terminated. Similarly, the feature ended considerably prior to US delivery in the serial compounds, but terminated concurrently with US delivery in simultaneous compounds. Furthermore, the A-US interval was equated across serial and simultaneous procedures, thus resulting in longer $X$-US intervals in serial procedures than in simultaneous procedures. Any of these differences between the serial and simultaneous compounds might have been responsible for the differences in learning observed with those procedures.

Experiments 1 and 2 examined the influence of several temporal variables on the acquisition of occasion setting, including the interval between the onset of the feature $(X)$ 
and the US, the interval between the onsets of $X$ and the common element $(A)$, the interval between the termination of $X$ and both the onset and termination of $A$, and the duration of $X$. Experiment 3 examined the extent of transfer of $X$ 's stimulus control after the various training procedures, in an attempt to specify the mechanism of action of the occasion setting observed in these experiments.

\section{EXPERIMENT 1}

Experiment 1 examined the influence of onset and termination asynchrony on the acquisition of occasion setting. Four groups of rats received feature-positive discrimination training with visual $X$ features and auditory $A$ common elements. The interval between $X$ onset and US delivery was identical in all four groups, but the durations of $X$ and $A$ were varied such that in one group (Group 15-15), $A$ and $X$ both started and terminated together (the simultaneous procedure), in Group 5-15, $A$ and $X$ started together, but $X$ ended prior to $A$ 's termination, in Group 15-5, $X$ started before $A$ but was coterminous with $A$, and in Group 5-5, $X$ both started and ended before $A$ was presented (the serial procedure that Ross and $I$ used). To examine transfer of occasion setting (reported in Experiment 3), all subjects were given training on two separate feature-positive discriminations, of identical temporal parameters.

\section{Method}

Subjects and Apparatus. The subjects were 18240 -day-old male and 14 210-day-old female albino rats. The rats were maintained at $80 \%$ of their ad-lib body weights throughout the experiment by limiting their access to food. They were housed individually in a colony that was illuminated between 6:00 a.m. and 8:00 p.m.

Eight experimental chambers, each $22.9 \times 20.3 \times 20.3 \mathrm{~cm}$, were used. The two end walls of each chamber were aluminum, and the side walls and the top were clear acrylic. A dimly illuminated food cup was recessed in the center of one end wall; a $6-\mathrm{W}$ jeweled panel light $6 \mathrm{~cm}$ above the food cup served as one feature CS. The chamber floors were made of $.48-\mathrm{cm}$ stainless steel rods spaced $1.9 \mathrm{~cm}$ apart. Each experimental chamber was enclosed in a soundresistant shell that contained speakers for the delivery of auditory stimuli and a normally off 6-W houselight, which served as the other feature CS. The houselight was located about $10 \mathrm{~cm}$ above and $10 \mathrm{~cm}$ in front of the front wall of the experimental chamber. The side wall of each shell contained an acrylic window to permit behavioral observations; above the window was a 6-W lamp within a red lens assembly, which provided dim general illumination. Two low-light television cameras were mounted $2.1 \mathrm{~m}$ from the experimental chambers so each could include four chambers in its field of view. Video-cassette recorders were programmed to record behaviors occurring during and 10-sec before and after CS presentations.

Behavioral observation procedures. All observations were made from videotapes. Each rat's behavior was observed at 1.25 -sec intervals during the 5-sec period immediately prior to CS presentations and during the CS presentations. The observations were paced by auditory signals recorded on the videotapes. On each observation, one and only one behavior was recorded. The measure percentage total behavior was calculated by dividing the number of instances of a particular behavior by the total number of observations made. Three behavioral categories were reported: rear, standing on hindlegs with both front feet off the floor; magazine, stand- ing motionless in front of the food magazine with head or nose within the recessed food cup; and head jerk, short rapid horizontal and/or vertical movements, usually (but not necessarily) directed toward the side of the chamber that contained the food cup (see Holland, 1977, for more complete descriptions).

Most observations were made by a single observer, but all test sessions and some randomly selected acquisition sessions were also scored by a second observer. The two observers agreed on $93 \%$ of their observations.

Procedure. All rats were first trained to eat from the recessed food cup. Twelve deliveries of two $45-\mathrm{mg}$ food pellets (the reinforcer used throughout these experiments) were given on a variabletime 1-min schedule. After $20 \mathrm{~min}$, the rats were removed from the chambers.

Then all rats received 27 90-min sessions of feature-positive discrimination training. All subjects were trained with two featurepositive discriminations. One of those discriminations comprised reinforced $X A+$ and nonreinforced $A-$ trials, and the other comprised reinforced $Y B+$ and nonreinforced $B$ - trials. The first 10 sessions contained two of each of those four kinds of trials; the remaining 17 sessions contained one $X A+$, one $Y B+$, three $A-$, and three $B-$ trials. The four kinds of trials were randomly intermixed in all sessions. For all subjects, the $X$ and $Y$ feature stimuli were the steady illumination of the houselight and the intermittent $(3 \mathrm{~Hz})$ illumination of the panelight, respectively, and the $A$ and $B$ commonelement stimuli were a steady white noise and a steady $1500 \mathrm{~Hz}$ tone, respectively.

Figure 1 portrays the temporal arrangements of cues within the reinforced compounds in the various groups during the discrimination phase. In all groups, the interval between the onset of the visual feature stimulus and food delivery was $15 \mathrm{sec}$ and the auditory common-element cue terminated simultaneously with food delivery. The groups were named to indicate first the duration of the visual features and then the duration of the auditory common elements. For example, the compound cues in Group 15-5 comprised 15-sec visual feature cues accompanied by auditory cues during the last $5 \mathrm{sec}$, followed immediately by food delivery; the nonreinforced cues were 5 -sec auditory stimuli. The auditory common element had the same duration on reinforced compound and nonreinforced common-element-alone trials.

\section{GROUP}

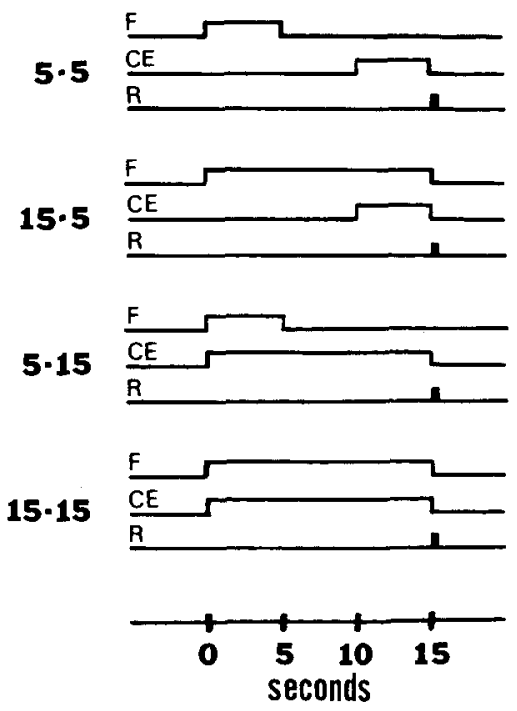

Figure 1. Arrangement of the feature (F), common-element (CE), and food reinforcer (R) cues during compound trials in Experiment 1. 


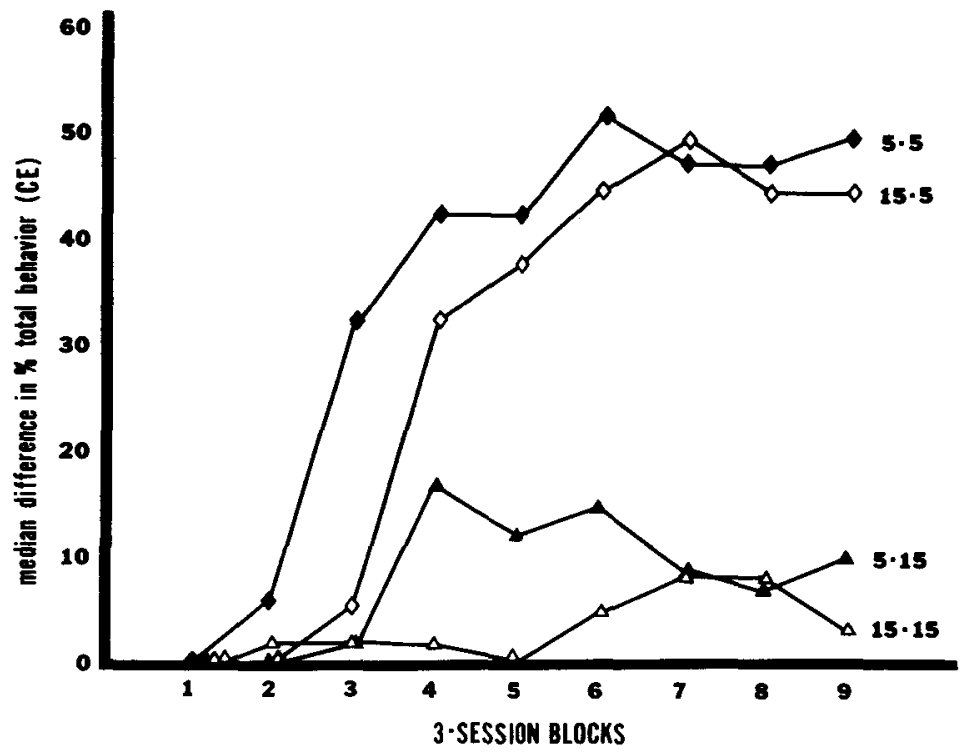

Figure 2. Acquisition of discriminated head-jerk behavior (responding during the common element on compound trials minus responding during commonelement-alone trials) in Experiment 1. The group names are indicated at the right of each curve.

\begin{abstract}
The measure of occasion setting was the difference in the frequency of head-jerk behavior to the auditory $A$ when it was presented within the $X A$ compounds and when it was presented alone. Distribution-free statistics were used, because of zero variances in several test conditions. Unless otherwise specified, the level of significance adopted was $p<.05$.
\end{abstract}

\section{Results and Discussion}

Figure 2 shows the acquisition of discriminated headjerk behavior in Experiment 1. The data from the two discriminations trained within each group were combined because those discriminations were acquired at similar rates in all groups. Asymptotically, there was substantial headjerk behavior during the auditory common elements on reinforced compound trials in the two groups in which the feature's onset preceded that of the common element (Groups 5-5 and 15-5), but only minimal levels of that behavior in the groups in which the onsets of the two cues were simultaneous (Groups 15-15 and 5-15). Over the final four three-session blocks, head-jerk behavior was reliably more frequent on reinforced than on nonreinforced trials in Groups 5-5 and 15-5 (Wilcoxon $T s=0$ ), but not in Groups 15-15 $(T=4$, four ties) or 5-15 ( $T=3$, four ties); the median frequency of head-jerk behavior on nonreinforced, element-alone trials was less than $1 \%$ in each of the four groups.

Distribution-free tests of analysis of variance hypotheses (Wilson, 1956), performed on both the difference scores and head-jerk behavior on reinforced compound trials, showed reliable effects of onset asynchrony $\left[x^{2} s(1)=32\right]$, but no effects of termination asynchrony and no interactions $\left[\chi^{2} s(1)=0\right]$. Subsequent multiple comparisons using Dunn's (1964) procedure (per experi- ment error rate $=.05$ ) showed that both the difference scores and reinforced compound responding were greater in both Group 5-5 and Group 15-5 than in either Group 5-15 or Group 15-15. Neither measure differed reliably between Groups 5-5 and 15-5 or between Groups 5-15 and 15-15. Thus, asymptotically, occasion setting was substantial when the onset of the auditory common element occurred after that of the visual feature, regardless of whether the feature ended prior to the onset of the common element or at the same time. Conversely, only minimal occasion setting occurred when the feature and common elements began together, regardless of whether the feature terminated before the common element terminated or at the same time.

However, the data from the early training sessions (first five three-session blocks) suggest that the acquisition of occasion setting may be enhanced when the termination of the feature precedes that of the common element. For both the difference scores and compound responding, Wilson (1956) tests showed reliable effects of both onset $\left[x^{2}(1)=17\right]$ and termination $\left[x^{2}(1)=4.5\right]$ asynchrony and a nonreliable interaction $\left[x^{2}(1)=0\right]$. Furthermore, Dunn's (1964) procedure showed that both measures were reliably greater in Group 5-5 (medians of $25 \%$ and $32 \%$, respectively) than in Group 15-5 (18\% and 22\%). Although Dunn's procedure showed no reliable differences between Group 5-15 (7\% and 12\%) and Group 15-15 (1\% and 3\%), less conservative Mann-Whitney $U$ tests showed marginally significant $(U s=16, p<.10$ ) superiority of the former group. Thus, although in these discrimination procedures, onset asynchrony of the feature and common element was the critical factor in determining the asymptotic performance of occasion setting, 
asynchrony of their termination influenced the acquisition rate.

The failure of the rats in Groups 15-15 and 5-15 to acquire discriminated head-jerk behavior does not reflect simply an inability to solve feature-positive discriminations with those intervals: those rats did learn the discriminations. Over the final four three-session blocks, Group $15-15$ showed $37 \%$ rear and $38 \%$ magazine behavior on compound trials, but only $4 \%$ rear $(T=0)$ and $16 \%$ magazine $(T=4)$ behavior on element-alone trials. Similarly, Group 5-15 showed $41 \%$ rear and $32 \%$ magazine behavior on compound trials, but only $9 \%$ rear $(T=0)$ and $21 \%$ magazine $(T=6, p<.10)$ behavior on element-alone trials. Nor can the greater occasion setting in Groups 5-5 and 15-5 than in Groups 15-15 and 5-15 be attributed solely to the shorter common-element duration and common-element-US interval in the latter groups. Ross and Holland (1981) found no evidence of occasion setting with either 5- or 10-sec simultaneous compounds. However, neither Ross and Holland's nor the present experiments comment on the possibility that commonelement duration and/or common-element-US interval influences occasion setting.

Finally, it might be argued that the between-groups differences in the frequency of head-jerk behavior were due solely to differences in simple conditioning of the auditory cues, rather than in occasion setting. That is, headjerk behavior may have been less frequent in Groups 515 and 15-15 than in Groups 5-5 and 15-5 simply because 15 -sec auditory cues paired with food generate less headjerk behavior than 5-sec cues, given equivalent numbers of training trials. Similarly, the concurrent presence of the visual cues at the time of reinforcement in Groups 15-5 and 15-15 may have produced more overshadowing of auditory-cue-food associations than occurred in Groups 5-5 and 5-15, respectively. Thus, equivalent occasionsetting abilities of the various features might still engender different levels of head-jerk behavior to the auditory comon elements. However, the differences in the levels of head-jerk behavior (Holland, 1980) and overshadowing (unpublished experiments) produced by these cues in nondiscriminative situations were not nearly large enough to account for the differences observed here. Thus, it seems more reasonable to attribute the differences observed here primarily to variations in occasion setting. Experiment 2 provides further evidence on this point.

\section{EXPERIMENT 2}

In Experiment 1, variations in the time of termination of the feature had relatively minor effects on occasion setting. Conversely, Ross and Holland (1981) suggested that the occurrence of a trace interval between the termination of the feature and the onset of the common element might be an important determinant of occasion setting, and noted that a procedure in which no such gap occurred (Ross \& Holland, Experiment 3, Group 5) generated considerably less evidence for occasion setting than did several other procedures in which there were gaps of various durations. However, the effects of such a gap were confounded with the interval between the onset of the feature and that of the common element and/or the US. It is possible that the relatively poor performance of Group 5 in Ross and Holland's (1981, Experiment 3) study was a function of the intervals involved, rather than of the lack of a trace interval between feature termination and the onset of the common element.

Experiment 2 examined the influence on occasion setting of the interval between feature onset and the common element and/or US, and of the time of termination of the feature. Three groups of rats received serial featurepositive discrimination training with a 10 -sec interval between the onset of the feature and the US (as in Ross \& Holland's no-gap procedure), and three groups received training with a 15-sec interval (as in Experiment 1, Groups 5-15 and 15-15). The common element was $5 \mathrm{sec}$ long in each group. The groups within each set of three differed in whether the feature terminated prior to the onset of the commmon element, coincident with that onset, or coincident with the termination of the common element.

In addition, Experiment 2 evaluated the possibility (raised in the discussion of Experiment 1) that variations in the amount of head-jerk behavior observed to the common-element cues in the different groups were due to differences in the strengths of common-element-US associations rather than in the amounts of occasion setting. Because, in Experiment 2, the common-element-US interval was identical in all groups, any variation in common-element-US associations across groups must be the consequence of differential overshadowing by the feature cues. Presumably, variations that would enhance the ability of a feature cue to overshadow commonelement-US associations would also enhance conditioning of that feature cue. Two measures of the strength of feature-US associations were taken in Experiment 2: (1) the ability of the feature stimuli to evoke conditioned responding in the absence of the common elements, and (2) the ability of the features alone to serve as reinforcers for the establishment of second-order conditioning to a novel cue (Holland, 1977).

\section{Method}

Subjects and Apparatus. The subjects were 40 male and $40 \mathrm{fe}-$ male experimentally naive albino rats, between 100 and 140 days old at the beginning of the experiment. They were maintained as described in Experiment 1.

The apparatus was the apparatus used in Experiment 1, plus another four chambers and sound-attenuating shells, which were very similar to the original eight chambers and shells.

Procedure. All subjects were first trained to eat from the food cup, as in Experiment 1. Then all rats received 16 sessions of serial feature-positive discrimination training. The first 4 sessions contained four reinforced compound trials and four nonreinforced common-element-alone trials; the last 12 sessions contained two reinforced and six nonreinforced trials. The feature cue was the intermittent $(3 \mathrm{~Hz}$ ) illumination of the houselight (of various durations in the different groups), and the common element was the 5-sec presentation of a $1500-\mathrm{Hz}$ tone. 
Figure 3 portrays the temporal arrangements of cues within the reinforced compounds in six of the seven groups of Experiment 2. In the seventh group, Group G-20, a 10-sec feature cue was followed, after a 5-sec gap, by the 5-sec common element. The groups were named to indicate, first, whether the termination of the feature (a) preceded a gap before the onset of the common element (GAP-groups), (b) occurred with the onset of the common element (ON-groups), or (c) was coincident with the termination of the common element (OFF-groups), and second, the interval between feature onset and the delivery of the US. For example, on compound trials in Group GAP-15, a 5-sec houselight feature was followed by a 5 -sec gap and then the $5-\mathrm{sec}$ tone common element.

Finally, the strength of the houselight feature cue was assessed in a second-order conditioning test. In each of three sessions, all subjects received eight trials that comprised a 5 -sec clicker $(8 \mathrm{~Hz})$, followed immediately by the houselight cue that was used in the previous phase. The duration of the houselight cue was the same as it was in the previous time. Behavior was recorded for $15 \mathrm{sec}$ after the onset of the houselight, regardless of its duration.

Experiment 2 was run in two shifts. One shift was conducted in the eight chambers used in Experiment 1; 8 subjects were run in each group. A second shift used four different, but similarly constructed, chambers. In that shift, 4 subjects were run in each group, except that no Group GAP-20 subjects were run. Thus, there were 12 subjects in each group, except for Group GAP-20, in which there were only 8 subjects. There were no other differences in either the experimental procedures or the outcomes of the two shifts.

\section{Results and Discussion}

Figure 4 shows the acquisition of discriminated headjerk behavior to the tone common elements, presumed to index occcasion setting. As in Experiment 1, there was little head-jerk behavior on common-element-alone trials ( $<4 \%$ overall in any group), so analyses of the difference scores were essentially identical to analyses of performances during the comon element on compound trials. Initially, a Wilson (1956) test was performed on the data from all groups except Group GAP-20. Those six groups formed a $2 \times 3$, feature-US interval $\times$ asynchrony type (gap, onset, or termination), factorial design. The effects of both feature-US interval $\left[\chi^{2}(1)=14.22\right]$ and asynchrony type $\left[\chi^{2}(2)=6.33\right]$ were reliable, but the interaction was not $\left[\chi^{2}(2)=0.78\right]$.

Further analyses were conducted using Dunn's (1964) multiple comparison procedure (per experiment error rate
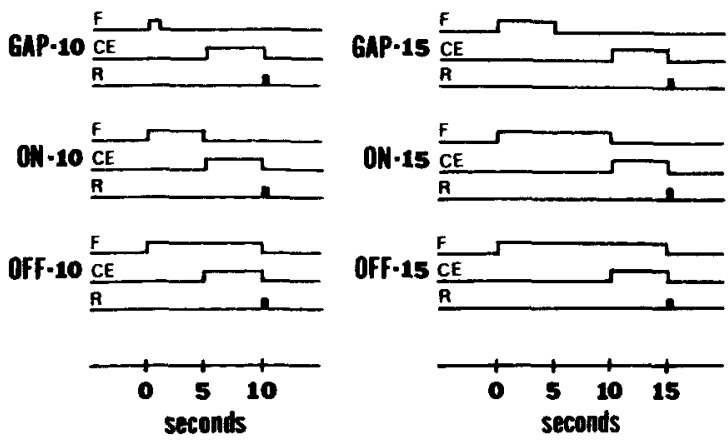

Figure 3. Arrangement of the houselight feature (F), the tone common element (CE), and the food reinforcer (R) on compound trials in Experiment 2. The arrangement of the stimuli in Group GAP20 is not shown (see text).

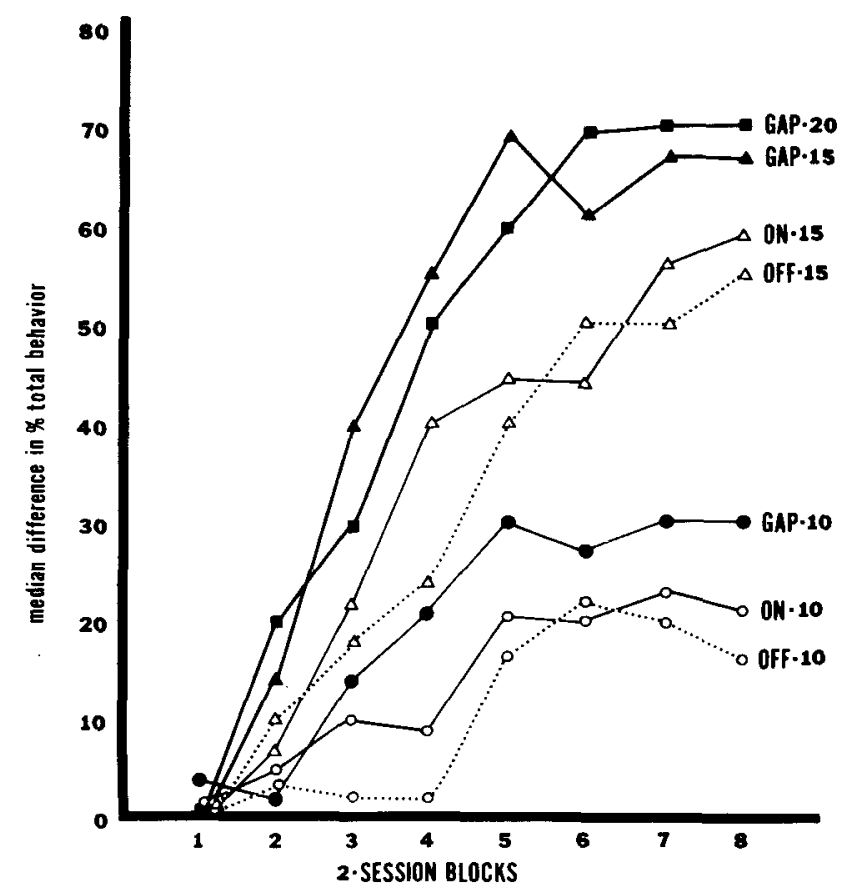

Figure 4. Acquisition of discriminated head-jerk behavior (responding during the common element on compound trails minus responding during common-element-alone trials) in Experiment 2. The group names are indicated at the right of each curve.

$=.05$ ) and individual Mann-Whitney $U$ tests. Unless otherwise noted, all differences described were reliable with both analyses. Consider first the effects of the interval between the onset of the feature and the delivery of the US. Each of the three groups that had 10-sec featureUS intervals (Groups GAP-10, ON-10, and OFF-10) showed reliably less discriminated head-jerk behavior than each of the groups that had 15-sec feature-US intervals (Groups GAP-15, ON-15, and OFF-15) and Group GAP20 , which had a 20 -sec feature-US interval $\left(U_{\mathrm{s}} \leq 22\right)$. Note that some of those comparisons were between groups that had equal feature durations but different termination arrangements (e.g., Groups ON-10 and GAP-15), and some were between groups that had identical termination arrangements but different feature durations (e.g., Groups $\mathrm{ON}-10$ and $\mathrm{ON}-15$ ). Thus, despite the confounding of feature-US interval with one or both of those variables in each separate comparison, the conclusion of greater occasion setting with the longer intervals is justified.

Next, consider the effects of the termination point of the feature stimulus. In Groups GAP-10, ON-10, and OFF-10, the feature-US interval was identical, but the feature terminated either before the onset or coincident with the onset, or was coterminous with the common element, respectively. Although Dunn's (1964) very conservative procedure showed no reliable differences among these three groups, Mann-Whitney $U$ tests showed that Group GAP-10 displayed more head-jerk behavior than either of the other two groups ( $U s \leq 34$ ), which did not differ 
from each other reliably $(U=47)$. Similarly, Group GAP-15 showed reliably more occasion setting than either Group ON-15 or Group OFF-15 (Us $\leq 27)$, which did not differ from each other $(U=61)$. Thus, as Ross and Holland (1981) suggested, the insertion of a gap between the termination of the feature and the onset of the common element enhanced occasion setting, with both 10 - and 15 -sec feature-US intervals.

The various feature termination points were confounded with the duration of the feature in each of the two triplets of groups just described. Groups GAP-20, ON-15, and OFF-10 all had 10-sec features, but differed in whether the feature terminated before the onset, coincident with the onset, or was coterminous with the common element, respectively. Group OFF-10 showed less discriminated head-jerk behavior than Group ON-15 $(U=15)$, which, in turn, showed more than Group GAP-20 $(U=31)$, although the latter difference was not reliable using Dunn's (1964) procedure. Of course, although equating feature duration, these comparisons are confounded with feature-US interval, which was already shown to be an important influence on occasion setting. However, it is worth noting that Ross and Holland (1981) found no effect of increasing the feature-US interval within the range of 15 to $30 \mathrm{sec}$. Consequently, one might argue that the difference between the performance of Groups GAP-20 and $\mathrm{ON}-15$ was relatively uninfluenced by feature-US interval. If so, then that comparison supports the claim that termination of the feature prior to the onset of the common element enhances occasion setting.

Table 1 shows the results of the final phase, which was designed to assess the strength of the visual features in the various groups. The left portion of the table shows rear and magazine behavior during each 5-sec period of the 15-sec observation interval that started with the onset of the visual cue. Session 1 data were selected because those data most accurately reflect performance to the visual cues at the end of training (e.g., that behavior had not yet begun to extinguish as a consequence of the non-

Table 1

Responding During Second-Order Conditioning Test of Experiment 2

\begin{tabular}{lllllllll} 
& \multicolumn{3}{c}{ Rear } & & \multicolumn{3}{c}{ Magazine } & HJK \\
\cline { 2 - 3 } \cline { 6 - 7 } Group & 1 & 2 & 3 & & 1 & 2 & 3 & 1 \\
\hline GAP-10 & 65 & 16 & 0 & 9 & 29 & 16 & 18 \\
ON-10 & $70^{*}$ & 20 & 0 & 11 & 31 & 21 & 17 \\
OFF-10 & $60^{*}$ & $35^{*}$ & 6 & & 12 & 26 & 19 & 26 \\
GAP-15 & $62^{*}$ & $15^{*}$ & 3 & 9 & 41 & 29 & 22 \\
ON-15 & $58^{*}$ & $31^{*}$ & 2 & 17 & 25 & 27 & 23 \\
OFF-15 & $51^{*}$ & $30^{*}$ & $11^{*}$ & & 19 & 31 & 38 & 24 \\
GAP-20 & $59^{*}$ & $26^{*}$ & 6 & 13 & 34 & 26 & 19 \\
\hline
\end{tabular}

Note-The rightmost column refers to head-jerk behavior during the clicker second-order conditioned stimulus. The other columns show rear and magazine behavior during each 5-sec period of the 5-sec observation interval that was initiated by the onset of the houselight reinforcer conditioned stimulus. Entries are median percentages of total behavior.

* signifies an interval that was filled with the houselight feature (included only for rear behavior). reinforced trials of this test procedure). Neither a KruskalWallis test on the data from all seven groups nor a Wilson test that excluded Group GAP-20's data (as described earlier) showed reliable differences among the groups for either behavior during any of the observation periods. The right side of Table 1 shows the frequency of head-jerk behavior during the second-order clicker CS, over all sessions. Kruskal-Wallis and Wilson tests indicated no differences among the groups. Thus, there was no evidence of reliable differences in the conditioning of the feature across the groups, despite substantial differences in the amount of discriminated head-jerk behavior to the auditory common-element cues. So it seems reasonable to attribute the latter differences to variations in occasion setting, rather than to differential overshadowing of common-element-US associations by the features.

In summary, discriminated head-jerk behavior to the auditory common-element cues was greater with 15 -sec feature-US intervals than with 10-sec intervals, and when a gap was inserted between the termination of the feature and the onset of the common element. Furthermore, there was no evidence that those differences in discriminated head-jerk behavior were due to differential overshadowing of common-element-US associations by the feature; thus, they presumably reflected variations in occasion setting.

\section{EXPERIMENT 3}

Experiment 3 examined the ability of an occasion setter established with one common element to augment responding evoked by other CSs. If occasion setting involves $X$ 's modulating the activity of a particular $A$-US association (as I suggested earlier), then it would not be anticipated that $X$ would set the occasion for responding to any cue except $A$. However, Bouton and Bolles (1985) have recently suggested that occasion setters may serve a disambiguating function, that is, they may select among multiple representations of events that have histories of both reinforcement and nonreinforcement. Similarly, Rescorla (1985) has suggested that occasion setters may act by lowering the threshold of activation of a representation of the US, such that an $A$-US association of otherwise subthreshold strength might generate CRs in their presence. Both of the latter notions suggest that the action of an occasion setter trained in an $X \rightarrow A+, A-$ serial feature-positive discrimination might extend to cues other than $A$.

In Experiment 3, the subjects of Experiment 1, which had received training on feature-positive discriminations of various temporal arrangements, were given transfer tests which assessed the ability of the feature cues to enhance responding evoked by (1) a cue that had served as the common element in another feature-positive discrimination, (2) a cue that had been first trained and then extinguished, and (3) a cue that had received only minimal amounts of training. 


\section{Method}

Subjects and Apparatus. The subjects and apparatus were those of Experiment 1.

Procedure. On the day immediately after the final discrimination session of Experiment 1, all rats received two transfer tests that examined each feature's ability to set the occasion for responding to its original common element and to the common element for the other feature-positive discrimination, and its ability to evoke conditioned responding in the absence of any common-element cue. Test 1 comprised two presentations each of houselight + noise and houselight + tone compounds, and of the houselight alone, and one presentation each of the tone and noise alone. Test 2 was identical to Test 1 except that the panelight was substituted for the houselight. No food was delivered in Tests 1 and 2. The temporal arrangements were those used in Experiment 1 The group nomenclature of Experiment 1 (Figure 1) was maintained in Experiment 3.

Half of the rats in each of the four groups were then given training designed to first condition and then extinguish responding to an 8-Hz clicker. The duration of the clicker in each group was identical to that of the other auditory cues. First, the rats received eight clicker-food pairings in each of four sessions. Then, after a day of retraining on the original discrimination task (identical to Session 1 of the discrimination training phase of Experiment 1), they received eight nonreinforced presentations of the clicker in each of two sessions. The other half of the rats received training designed to establish to the clicker conditioned responding of similar magnitude, but with no history of nonreinforcement. They received only the first two clicker-food sessions and the original discrimination retraining session. On the other four sessions of this phase, they remained in their home cages.

Then, all rats received two test sessions to assess the ability of each feature to set the occasion for responding to the clicker cue. Test 3 comprised two presentations each of the houselight + noise, houselight + clicker, and houselight cues, and one presentation each of the noise and the clicker. Test 4 comprised two presentations each of the panelight + tone, panelight + clicker, and panelightalone cues, and one presentation each of the tone and the clicker. No food was delivered in Tests 3 and 4.

Finally, the relative contributions of learning and performance factors in generating the various performances were examined. In these tests, the rats in Group 5-5 were tested with the training stimulus parameters of Group 15-5, and vice versa. Similarly, the training and testing parameters of Groups 15-15 and 5-15 were swapped. Initially, a single day of retraining of the two feature-positive discriminations was followed by two test sessions identical to Tests 1 and 2 (except for the temporal parameters). However, the response levels in those sessions were very low, so a second set of tests, identical to the first set, was given after six retraining sessions. Only the data from these final tests (Tests 5 and 6) are presented below.

\section{Results and Discussion}

Figure 5 shows head-jerk behavior during Tests 1-4. Performance on the original discriminations was similar during Tests 1 and 2 and Tests 3 and 4; those data are combined in Figure 5 for ease of comparison (left two bars in each panel). All of the subjects in Groups 5-5, 15-5, and 5-15 and 5 of the 8 subjects in Group 15-15 responded more to the $A$ and $B$ cues when they were preceded by their original feature cues than when they were presented alone. Because none of the subjects responded to either $\boldsymbol{A}$ or $\boldsymbol{B}$ when those cues were presented alone, only responding during the compounds is analyzed below. As in the discrimination phase of Experiment 1 (Figure 2), onset asynchrony was an important deter-
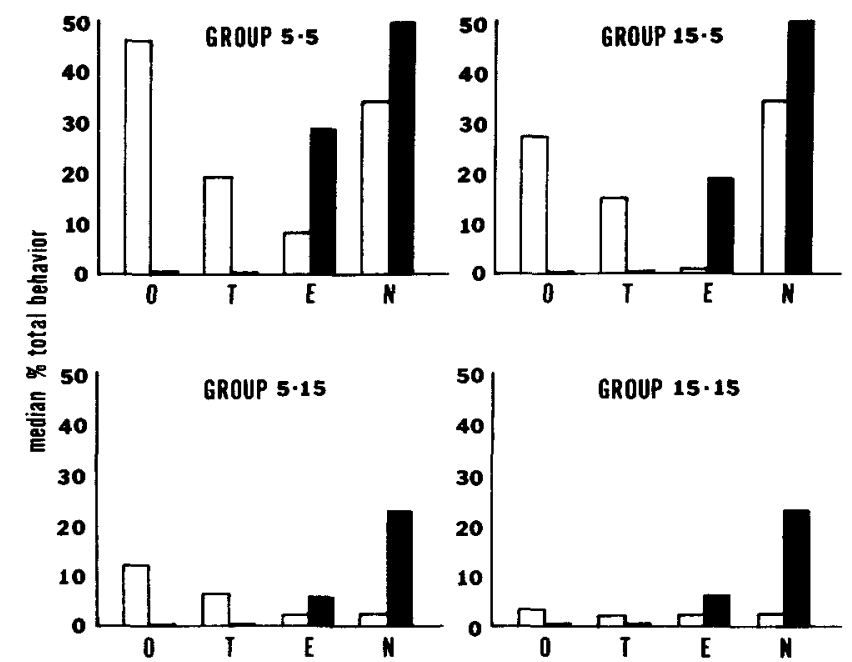

Figure 5. Head-jerk behavior during Tests 1-4 of Experiment 3 . The open bars show responding during the target cue on compound trials, and the shaded bars indicate responding to that torget when it was presented alone. The letters on the abscissas indicate the nature of the target cue: $\mathbf{O}=$ original common element, $T=c o m-$ mon element from the other feature-positive discrimination, $\mathbf{E}=$ trained and extinguished stimulus, $\mathbf{N}=$ weakly trained stimulus.

minant of occasion setting: there was a reliable effect of that variable $\left[\chi^{2}(1)=16\right]$, and comparisons with Dunn's (1964) procedure showed that head-jerk behavior during the auditory cues on compound trials was reliably more frequent in Groups 5-5 and 15-5 than in each of the other two groups. However, in support of the early acquisition data of Experiment 1 and the data of Experiment 2, these data indicated that termination asynchrony also influenced occasion setting. More occasion setting was observed in the groups in which the visual features terminated prior to the termination of the auditory common elements $\left[x^{2}(1)=4\right]$. Individual comparisons indicated that Group 5-15 showed more head-jerk behavior than Group 15-15, but Group 5-5 showed only marginally more responding than Group 15-5, even with a Mann-Whitney $U$ test $[U=18, p<.10]$.

The primary purpose of Tests 1-4 was to examine the features' abilities to set the occasion for head-jerk responding to excitors other than the ones with which they were originally trained. Consider first the two groups (top panels of Figure 5) that showed substantial amounts of occasion setting within the original training compounds. Both Group 5-5 and Group 15-5 showed reliable transfer of the features' ability to set the occasion for responding to the common element that was involved in the other feature-positive discrimination. That is, responding to $A$ and $B$ was more frequent on $X B$ and $Y A$ trials than on $A$ and $B$-alone trials $(T s=0)$. That transfer was more substantial in Group 15-5 than in Group 5-5. Responding during presentations of the original, $X \boldsymbol{A}$ and $Y B$, compounds was reliably greater than that during presentations of the transfer, $X B$ and $Y A$, compounds in Group 5-5 $(T=0)$, 
but not in Group 15-5 $(T=11.5)$. Furthermore, the difference in the frequency of responding on the original and transfer compounds was reliably greater in Group 5-5 than in Group 15-5 ( $U=7.5)$.

Conversely, the features showed no ability to set the occasion for responding to either the trained and extinguished or weakly trained excitors that had not been trained within a feature-positive discrimination. In fact, in both Group 5-5 and Group 15-5, the prior presentation of the visual features suppressed behavior to the trained and exstinguished cue in all subjects. Similarly, the features suppressed responding to the weakly trained excitor in all of the subjects in Group 15-5 and in half of the subjects in Group 5-5. That suppression, in addition to denying the occurrence of transfer of occasion setting to these kinds of excitors, shows that the display of head jerk during the common elements within the compounds but not during the elements alone is not merely the consequence of the summation of subthreshold response tendencies conditioned to the feature and common-element cues. Finally, the level of head-jerk behavior that occurred on feature-alone trials, during the interval in which a common element would normally have been presented, was $1 \%$ in Group $5-5$ and $0 \%$ in Group 15-5.

Lower levels of occasion setting were observed in Group 5-15 (lower left panel of Figure 5). Nevertheless, as in Groups 5-5 and 15-5, there was reliable transfer of an occasion setter's ability to modulate responding to the cue that had been used as the common element in the other feature-positive discrimination $(T=0)$, but no transfer to a trained and extinguished cue, and suppression of responding to a weakly trained cue. There was no evidence of discriminative head-jerk behavior in Group 1515 , except that presentation of the feature suppressed head-jerk behavior evoked by the weakly trained cue in all subjects.

After retraining of the original two feature-positive discriminations in all groups, responding to the $X A, X B, Y A$, and $Y B$ compounds, as well as to $A, B, X$, and $Y$ alone, was again examined. However, in those tests (Tests 5 and 6 ), the temporal arrangements of the compounds were switched so that, in Group 5-5, the compounds were presented as they had been in training in Group 15-5, and vice versa. Similarly, the arrangements of cues in Groups 5-15 and 15-15 were switched. In Group 5-5, head-jerk behavior comprised $33 \%$ of the total behavior during the common element within the original compounds and $13 \%$ during the transfer compounds. In Group 15-5, head-jerk behavior comprised $22 \%$ of the behavior during the original compounds and $13 \%$ on the transfer compounds. As in Tests 1 and 2 , responding on the original compounds was greater in Group 5-5 $(U=18, p<.10)$. Also, as in Test 1, the differences between responding on the original and transfer compounds were greater in Group 5-5 $(U=11)$. Thus, both the amount of responding to the original compounds and the relative amounts of transfer in these two groups were functions of the temporal parameters of training, not testing. There was no evidence for occasion setting in Groups 5-15 and 15-15 in Tests 5 and 6.

\section{GENERAL DISCUSSION}

As in Ross and Holland's (1981) experiments, the form of the CRs that occurred in feature-positive discriminations depended on the temporal arrangements of the cues within the reinforced compound. In simultaneous featurepositive discriminations (Group 15-15 of Experiment 1), the visual feature stimuli acquired rear and magazine CRs, which indicated that the feature was associated with the US. But those features acquired little ability to modulate the occurrence of head-jerk behavior to the auditory common elements. In discriminations that involved nonsimultaneous compounds, however, the visual feature acquired the ability to set the occasion for head-jerk behavior to the auditory common elements. Three contributors to the emergence of occasion setting were identified. First was the relation of the onsets of the feature and commonelement cues. The most substantial occasion setting occurred when the onset of the visual feature occurred before the onset of the auditory common element (Groups 5-5 and 15-5 of Experiment 1, and all of the groups of Experiment 2). Second, longer feature-US intervals encouraged occasion setting, within the bounds of the parameters examined here (Experiment 2). Finally, the time of the termination of the feature appeared to contribute to the magnitude of occasion setting. Small amounts of occasion setting were observed when the feature terminated before the end of the common element, even when the two cues had simultaneous onsets (Group 5-15 of Experiment 1), and the occasion setting observed with asynchronous onsets was enhanced if the feature terminated before the onset of the common element (Group 5-5 of Experiments 1 and 3, and Groups GAP-10, GAP15, and GAP-20 of Experiment 2).

A casual description that fits the findings of these experiments is that occasion setting is encouraged whenever a perceptual discontinuity in the stream of events occurs (e.g., Estes, 1979). Simultaneous presentation of a light and a tone followed by food might encourage the subject to abstract light-food, tone-food, and perhaps configural "light + tone"-food relations, whereas serial light-thentone-then-food presentations might encourage the encoding of the relation between the light and the tone-food relation. The insertion of trace intervals or arrangement of other asynchronicities between the onsets and terminations of the feature and common-element cues further segments the sequence of events, and thus further encourages occasion setting. Within this perspective, it might be anticipated that the intervals, and onset and termination asynchronies between the common element and the US, as well as the relations between the intervals and asynchronies among the various events, would also affect occasion setting.

It is worth speculating that other forms of perceptual discontinuity in the event sequence might also encourage occasion setting. For example, Holland (1983) found that 
occasion setting was acquired more rapidly if the feature and common-element cues were of different modalities (one auditory and one visual) than if they were of the same modality (see also Moore et al., 1969). Other stimulustype contrasts, for example, short versus long, discrete versus diffuse (e.g., Jenkins, 1985; Rescorla, 1985), or explicit versus contextual (e.g., Balaz, Capra, Hartl, \& Miller, 1981; Bouton \& Bolles, 1985) might similarly encourage the engaging of a more hierarchical coding of sequence information (e.g., Fountain, Henne, \& Hulse, 1984). Furthermore, the contrast of place and event (e.g., Hirsh, 1980; Nadel \& Willner, 1980) and the spatial discontinuities encountered as an organism ranges over a variety of food locations (e.g., Olton, Becker, \& Handelmann, 1979) might especially encourage occasion setting.

The feature cues were effective in setting the occasion for responding not only to the common elements with which they were trained, but also to stimuli that were trained as common elements within other feature-positive discriminations (Experiment 3). However, they either suppressed or had no effect on the head-jerk behavior evoked by either trained and extinguished or weakly trained excitors that had not been part of the featurepositive discriminations.

The implications of these transfer data are unclear. At face value, they are damaging to all of the notions about the action of occasion setters that I described earlier: Both Bouton and Bolles (1985) and Rescorla (1985) anticipate facilitation of responding to the trained and extinguished cue as well as to the other common element, whereas I (Holland, 1983, 1985) expect no transfer in either case. However, interpretation of these data is clouded by several factors. First, it is possible that the differential transfer to the other common element and the trained and extinguished cue was related to the differential similarity of the testing context to the various training contexts. Both $X A+, A-$ and $Y B+, B-$ trials were intermixed throughout training, as in testing, whereas training of the conditioned and extinguished cue was massed, in the absence of trials from either discrimination. It is unlikely, though, that this factor accounts entirely for the differential transfer: Elsewhere (Holland, in press), I have found no evidence of transfer to trained and extinguished cues after training in which the conditioning and extinction presentations of an excitor were intermixed with feature-positive discrimination trials like those received by Group 5-5.

Second, transfer to the other common element might simply reflect generalization or confusion between the two visual features in training or testing. Other, unpublished, data from my laboratory show substantial generalization of simple CRs between the two visual cues used here. Thus, stimulus elements common to $X$ and $Y$ would serve as occasion setters for both $A$ and $B$, and transfer would be anticipated even if occasion setters activated specific CS-US associations, as I have claimed. Thus, transfer of occasion-setting powers under these circumstances is a convincing disconfirmation of my claim for specificity of that function only to the extent that the transfer is greater than might be anticipated on the basis of the known amounts of generalization among the cues. Note that because no stimulus elements of either $X$ or $Y$ were ever presented in a conditional relation with the trained and extinguished cue, confusion between $X$ and $Y$ would be irrelevant to any responding observed to that cue. Consequently, transfer to such a cue would be anticipated to occur only to the extent that it shares features with the common elements. Because I typically find little or no generalization of CRs among the three auditory cues used here, the lack of transfer observed here is not surprising.

One feature of these data seems inconsistent with the possibility that the transfer to the other common element was due solely to simple generalization. It seems reasonable to expect that the traces or memories of two cues would be more difficult to distinguish than those two cues themselves. Thus, on the basis of generalization, one might expect greater transfer in Group 5-5, in which the feature cue was terminated $5 \mathrm{sec}$ prior to the onset of the common element, than in Group 15-5, in which the feature cue was present during the common element. However, relatively greater transfer to the other common element was observed in Group 15-5. That difference might reflect nothing more than scaling or floor effects. But it is worth noting that Davidson and Rescorla (in press) reported substantial transfer in a related conditioning situation when those temporal parameters were used. Although a disturbing possibility, perhaps different arrangements of cues within serial compounds encourage different learning, just as I have reported that simultaneous and serial compounds generate different learning.

\section{REFERENCES}

Balaz, M. A., Capra, S., Hartl, P., \& Miller, R. R. (1981). Contextual potentiation of acquired behavior after devaluing direct contextUS associations. Learning \& Motivation, 12, 383-397.

Bouton, M. E., \& Bolles, R. C. (1985). Contexts, event memories, and extinction. In P. Balsam \& A. Tomie (Eds.), Context and learning. Hillsdale, NJ: Erlbaum.

Davidson, T. L., \& REscoRla, R. A. (in press). Transfer of facilitation in the rat. Animal Learning \& Behavior.

DuNN, O. J. (1964). Multiple comparisons using rank sums. Technometrics, 6, 241-252.

EsTES, W. K. (1979). Cognitive processes in conditioning. In A. Dickinson \& R. A. Boakes (Eds.), Mechanisms of learning and motivation (pp. 417-441). Hillsdale, NJ: Erlbaum.

Fountain, S. B., Henne, D. R., \& Hulse, S. H. (1984). Phrasing cues and hierarchical organization of serial pattern learning by rats. Journal of Experimental Psychology: Animal Behavior Processes, 10, $30-45$.

HiRsh, R. (1980). The hippocampus, conditional operations, and cognition. Physiological Psychology, 8, 175-182.

Holland, P. C. (1977). Conditioned stimulus as a determinant of the form of the Pavlovian conditioned response. Journal of Experimental Psychology: Animal Behavior Processes, 3, 77-104.

HollaNd, P. C. (1980). CS-US interval as a determinant of the form of Pavlovian appetitive conditioned responses. Joumal of Experimental Psychology: Animal Behavior Processes, 6, 155-174.

Holland, P. C. (1983). Occasion setting in Pavlovian feature positive 
discriminations. In M. L. Commons, R. J. Herrnstein, \& A. R. Wagner (Eds.), Quantitative analyses of behavior: Discrimination processes (Vol. 4, pp. 183-206). New York: Ballinger.

Holland, P. C. (1985). The nature of conditioned inhibition in serial and simultaneous feature negative discriminations. In R. R. Miller \& N. E. Spear (Eds.), Information processing in animals: Conditioned inhibition (pp. 267-297). Hillsdale, NJ: Erlbaum.

Holland, P. C. (in press). Transfer after serial feature positive discrimination training. Learning \& Motivation.

JENKINS, H. M. (1985). Conditioned inhibition of key pecking in the pigeon. In R. R. Miller \& N. E. Spear (Eds.), Information processing in animals: Conditioned inhibition (pp. 327-353). Hillsdale, NJ: Erlbaum.

Jenkins, H. M., \& SaINSBURy, R. S. (1969). The development of stimulus control through differential reinforcement. In N. J. Mackintosh \& W. K. Honig (Eds.), Fundamental issues in associative learning (pp. 123-161). Halifax, N.S.: Dalhousie University Press.

Moore, J. W., Newman, F. L., \& Glasgow, B. (1969). Intertrial cues as discriminative stimuli in human eyelid conditioning. Journal of $E x$ perimental Psychology, 79, 319-326.
NADEl, L., \& Willner, J. (1980). Context and conditioning: A place for space. Physiological Psychology, 8, 218-228.

Olton, D., Becker, J. T., \& Handelmann, G. E. (1979). Hippocampus, space, and memory. Behavioral \& Brain Science, 2, 313-365.

Rescorla, R. A. (1985). Inhibition and facilitation. In R. R. Miller \& N. E. Spear (Eds..), Information processing in animals: Conditioned inhibition (pp. 299-326). Hillsdale, NJ: Erlbaum.

Ross, R. T., \& Holland, P. C. (1981). Conditioning of simultaneous and serial feature positive discriminations. Animal Learning \& $\mathrm{Be}$ havior, 9, 293-303.

SkINNER, B. F. (1938). The behavior of organisms. New York: Appleton-Century.

WILSON, K. V. (1956). A distribution-free test of analysis of variance hypotheses. Psychological Bulletin, 53, 96-101.

(Manuscript received November 5, 1985; revision accepted for publication March 18, 1986.) 